\title{
Lighting up Electrochemiluminescent Inactive Dyes by Intramolecular Resonance Energy Transfer
}

Yongjun Zheng, Hong Yang, Lufang Zhao, Yuhan Bai, Xinhua Chen, Kaiqing Wu, Songqin Liu, Yanfei Shen, Yuanjian Zhang*

Medical School, School of Chemistry and Chemical Engineering, Southeast University, Nanjing 210009, China.

E-mail: Yuanjian.Zhang@seu.edu.cn

\begin{abstract}
:
By virtue of near-zero optical background and photobleaching, electrochemiluminescence (ECL), an optical phenomenon excited by electrochemical reactions, has drawn extensive attention in both fundamental studies and wide applications especially of ultrasensitive bioassay. Developing diverse ECL emitters is crucial to unlock their multiformity and performances, but remains a formidable challenge, due to the rigorous requirements for ECL. Herein, we report a general intramolecular ECL resonance energy transfer (iECL-RET) strategy to light up ECLinactive dyes in aqueous solutions using an existing high-performance ECL initiators. As a proof-of-concept, a series of luminol donor-dye acceptor based ECL emitters with near unity RET efficiency and coarse/fine tunable emission wavelengths were demonstrated. Different to previous exploitation of new molecule single-handedly to address all the prerequisites of ECL, each unit in the proposed ECL ensemble performed maximally its own functions. The iECL-RET strategy would greatly expand the family members of ECL emitters for more demanding future applications.
\end{abstract}

Keywords: electrochemiluminescence, electrochemiluminescent emitters, fluorescence dyes, resonance energy transfer 


\section{Introduction}

Due to near-zero background optical noises and photobleaching, electrochemiluminescence (ECL) is widely used to pursue the ultimate analytical limit in diverse fields ranging from clinical diagnosis, food analysis, environmental monitoring to bioimaging since the pioneering works in the mid-1960s. ${ }^{1-5}$ As one of the most essential criteria, ECL emitter directly determines the strategies and performances of application methods. ${ }^{6,7}$ For this, developing new emitters has been one of the forefronts of ECL-based research, but remains a formidable challenge, even by using dyes with strong fluorescence (FL). In principle, for the common co-reactant mechanism-based ECL, there are three essential prerequisites for an efficient emitter (Figure 1a). ${ }^{8,9}$ Firstly, ECL emitters should be ready to exchange high-energy electrons with working electrodes. Secondly, ECL emitters in high energy state could react with a co-reagent radical and generate excitation state, during which self-annihilation or quenching by ambient molecules in aqueous solution are not evident. Lastly, the radiative transition should dominate the final recombination of excitons. Generally, it is hard to meet all these requirements (Figure 1b), making the type of ECL active molecules be limited.

Current available ECL emitters mainly include metal complexes, ${ }^{10-12}$ semiconducting nanoparticles/nanoclusters, ${ }^{13-16}$ conjugated organic molecules ${ }^{17-19}$. Great efforts of modulating electron transfer among electrode, emitters and/or co-reactants by potential control, ${ }^{20-24}$ aggregation-induction, ${ }^{25-29}$ self-enhancement, ${ }^{30-34}$ crystallization, ${ }^{35-38}$ as well as other strategies, ${ }^{39-42}$ have been devoted to improving ECL efficiency and adapting to different application schemes. Despite these unprecedented success in mechanism studies and some applications, ${ }^{43-46}$ developing ECL emitters with reasonable ECL efficiency, good water-solubility/-dispersibility, and facile being labeled is still in infancy. For instance, only tris(2,2'-bipyridine)ruthenium(II) $\left(\left[\mathrm{Ru}(\mathrm{bpy})_{3}\right]^{2+}\right)$ has been used in commercialized ECL system, which significantly hampers their prospective applications. Therefore, there is an increasing demand for 
developing diverse ECL emitters to unlock their multiformity and performances.

Rather than rigorous designing a totally new molecule that addressed all the prerequisites for ECL in previous reports, ${ }^{47,48}$ we reasoned that if it was possible to create ECL ensemble, in which each unit performed its own functions. For instance, resonance energy transfer (RET) is a mature strategy to give materials new functions and innovating applications, mediated by electronic dipole-dipole coupling to induce non-radiative transfer of electronic excitations from donor to acceptor. ${ }^{49-51}$ Lighting up inactive ECL dyes by RET from an existing high-performance ECL initiators would be an intriguing general way to prepare new ECL emitters with variable properties. Despite there have been biosensing investigations regarding RET between molecular recognition-triggered ECL donor-accepter pairs for sensitivity enhancement, ${ }^{52-54}$ development of new ECL emitters using RET has not been reported.

(a) Active ECL pathways

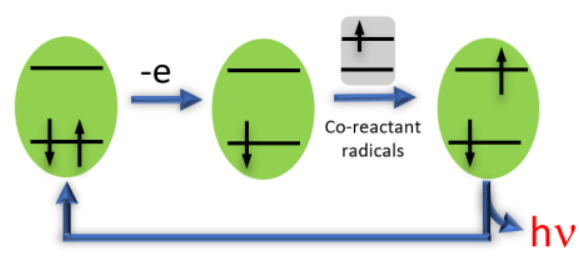

(b) Inactive ECL pathways

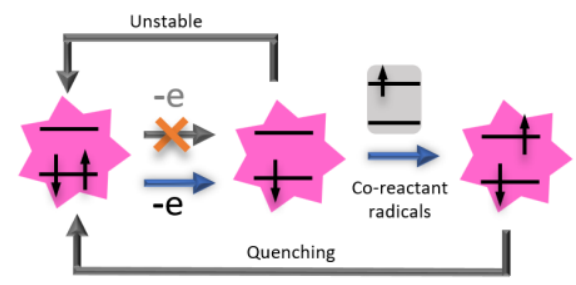

(c) ERET-ECL pathways (this work)
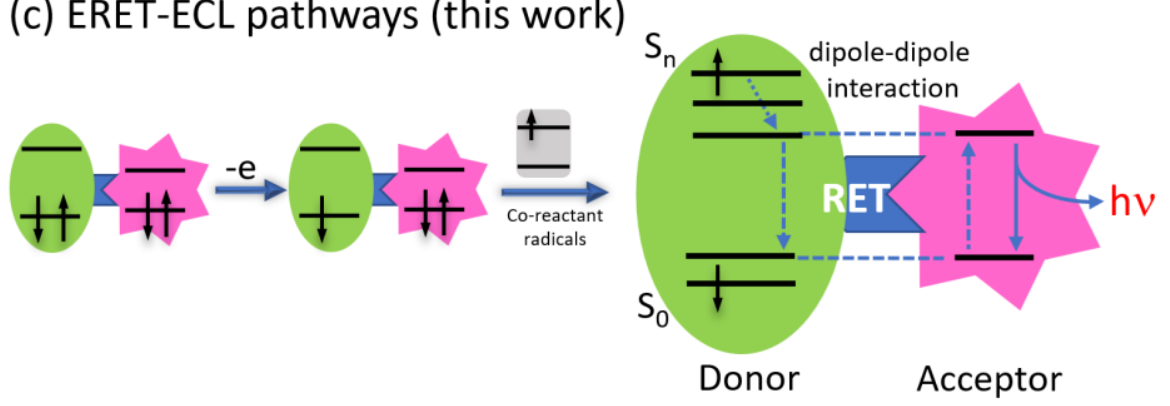

(d)<smiles>CCN(CC)c1ccc2cc(C(=O)N3CCN(CC(=O)Nc4cccc5c(=O)[nH][nH]c(=O)c45)CC3)c(=O)oc2c1</smiles>

LU-CO 
Figure 1 Design of lighting up inactive ECL emitters by iECL-RET. Brief mechanism of ECL active (a) and inactive (b) emitters, and (c) the proposed iECL-RET activation of ECL-inactive emitters. For clarity, only anodic ECL processes are shown. (d) Two examples of iECL-RET-based ECL emitters.

Here, we report a general intramolecular ECL resonance energy transfer (iECL-RET) strategy to light up ECL-inactive dyes in aqueous solutions (Figure 1c). As a proof of concept, two classical dyes, i.e., (7-(Diethylamino) coumarin-3-carboxylic acid (CO) and 3-(4-amino-1,8-naphthalimido) propanoic acid (NA) that are ECL inactive, were demonstrated to be lighted up using covalently linked luminol as an ECL initiator with a RET efficiency of near-unity (Figure 1d). Moreover, modification of electronwithdrawing substituents on dyes further fine-tuned the ECL emission wavelength.

\section{Results and Discussion}

For a highly efficient iECL-RET, the choosing of the donor, acceptor, and linker is crucial. As a proof of principle, luminol (LU) with high ECL activity was used as the donor. Considering the ECL emission wavelength of luminol and the absorption wavelength of FL luminophores, $\mathbf{C O}$ and NA were selected as the two-model acceptors. As shown in Figure 2a and $\mathbf{2 b}$, the overlap between the emission of luminol and the absorption of $\mathbf{C O}$ and NA were profound. The quantitative analysis indicated the spectral overlap integrals $(J)$ was up to $6.46 \times 10^{14} \mathrm{~nm}^{4} \mathrm{M}^{-1} \mathrm{~cm}^{-1}$ and $6.83 \times 10^{14} \mathrm{~nm}^{4} \mathrm{M}^{-}$ ${ }^{1} \mathrm{~cm}^{-1}$, respectively. Complementarily, the frontier orbitals energies of HOMOs and LUMOs of LU, CO, and NA were also calculated by density functional theory (DFT). As shown in Figure 2c and S1, the HOMO-LUMO energy levels were adequately aligned, allowing energy transfer between $\mathbf{L U}$ and $\mathbf{C O}$ or NA. Therefore, the experimental spectra and theoretical calculation suggested that $\mathbf{C O}$ and NA are two ideal energy acceptors for the luminol donor. 
(a)

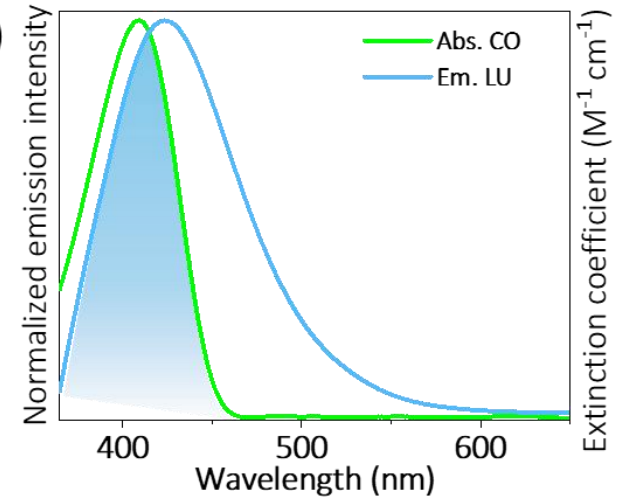

(b)

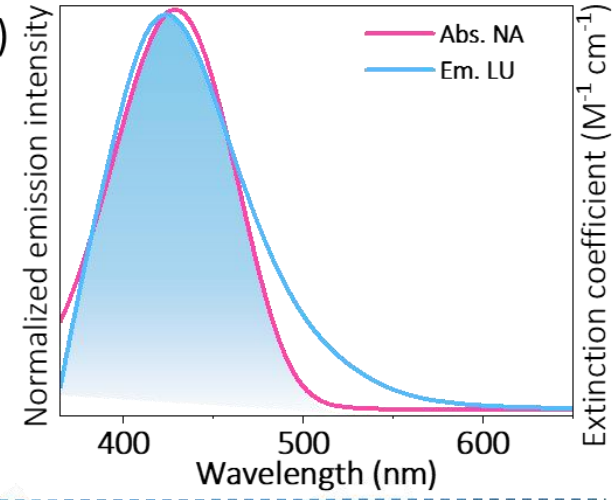

(c)
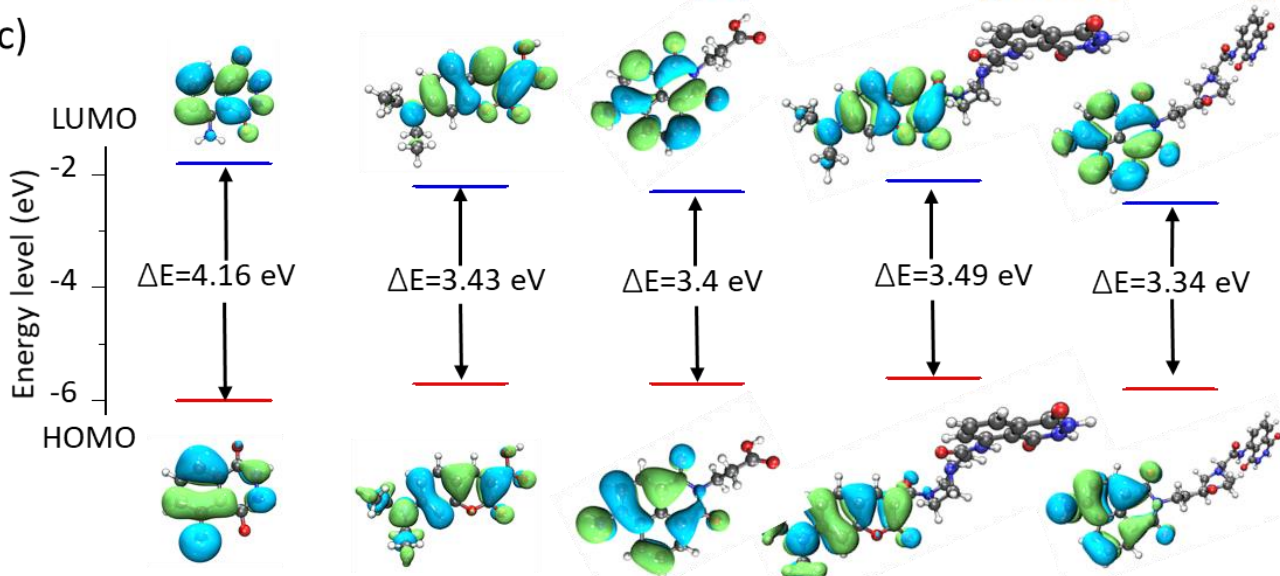

LU

$\mathrm{CO}$

LU-CO

LU-NA

(d)

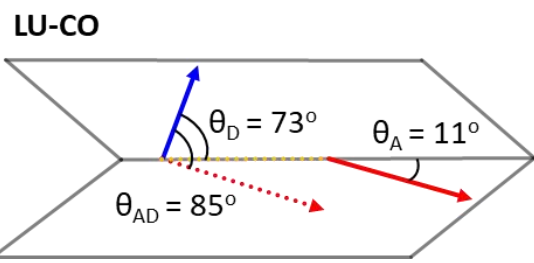

LU-NA

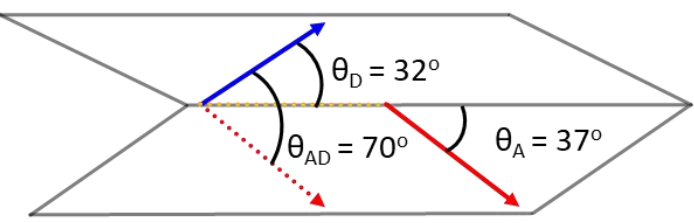

Figure 2. Principle validation of RET between LU and CO or NA. Absorption spectra (Abs.) of CO (a, green line), NA (b, pink line) and normalized ECL emission spectra (Em.) of luminol ( $\mathrm{a}, \mathrm{b}$, blue line) in PBS $(\mathrm{pH}=8.0,0.01 \mathrm{M})$. Blue area: the spectral overlap integrand. Electronic isodensity plots and frontier orbitals energies (c) of HOMOs and LUMOs of LU, CO, NA, LU-CO, and LU-NA based on the DFT/b3lyp/6-311+g(d). Chart of dipole angles with the center-to-center vector of the LU-CO and LU-NA (d). Blue: transition dipole moments of donor emission. Red: the acceptor absorption. Yellow: the connecting vector. $\theta_{\mathrm{DA}}$ : the angle between donor emission and acceptor absorption dipole moments. $\theta_{\mathrm{D}}$ and $\theta_{\mathrm{A}}$ : the angles between the respective dipole moments.

Appropriate distance and angle between $\mathbf{L U}$ and $\mathbf{C O}$ or NA are another critical factor 
for effective iECL-RET. For this, a piperazine derivative was chosen as the linker to conjugate LU and CO/NA because piperazine has an advantage in self-adjustable conformations. ${ }^{55,56}$ Along this line, the structures of the targeted LU-CO and LU-NA molecules were firstly optimized by DFT calculation (Figure S1). The electronic isodensity plots and frontier orbitals energies of LU-CO and LU-NA were obtained by DFT and time dependent DFT (TD-DFT). As shown in Figure 2c and Table S1, the HOMO-LUMO gap of LU-CO and LU-NA were much smaller than the $\mathbf{L U}$ monomer and close to the corresponding dye monomer. In addition, the electrons were mainly distributed in the dye unit, indicative of effective donor-acceptor interaction with $\mathbf{L} \mathbf{U}$ and the potential domain in charge of the final ECL emission. The core-to-core distance (R) between LU and the dyes in LU-CO or LU-NA were calculated as $11 \AA$ and $16 \AA$, respectively (Figure S2), which is in line with the distance rule of RET. ${ }^{57,58}$ Moreover, the dipole transitions moments and the angles with the center-to-center vector of $\mathbf{L U}$ CO and LU-NA were also calculated. As shown in Figure 2d \& S3, the orientation factor $\left(\kappa^{2}\right)$ of $\mathbf{L U}-\mathbf{C O}$ and $\mathbf{L U}-\mathbf{N A}$ were calculated as 0.59 and 2.89, respectively, which were also within the reasonable range $\left(0 \leq \kappa^{2} \leq 4\right) .{ }^{58}$ Accordingly, the critical Förster radius $\left(\mathrm{R}_{0}\right)$ of $\mathbf{L U}-\mathbf{C O}$ and $\mathbf{L U}-\mathbf{N A}$ was evaluated to be $27 \AA$ and $35 \AA$, respectively, indicative of a very promising RET efficiency $\left(\Phi_{\mathrm{RET}}\right)$ by taking the practical lower R into account. ${ }^{57,58}$ Therefore, principally, the proposed LU-CO and LU-NA meet the requirements of RET.

Guided by the above theoretical prediction, LU-CO and LU-NA were synthesized from luminol and dyes (Figure 3a) using piperazine derivative as the bridge. Since carboxyl group is a typical structure in most organic dyes, in order to make the synthetic routes general, the intermediate product, i.e., amino group-terminated luminol (LUNH), was first synthesized. Consequently, LU-CO and LU-NA were obtained by a facile amidation reaction between dyes and LU-NH with a total yield of ca. 37\%. The structures of all compounds were confirmed by ${ }^{1} \mathrm{H}-\mathrm{NMR},{ }^{13} \mathrm{C}-\mathrm{NMR}$, and highresolution mass spectrum (Figure S4-S12). 

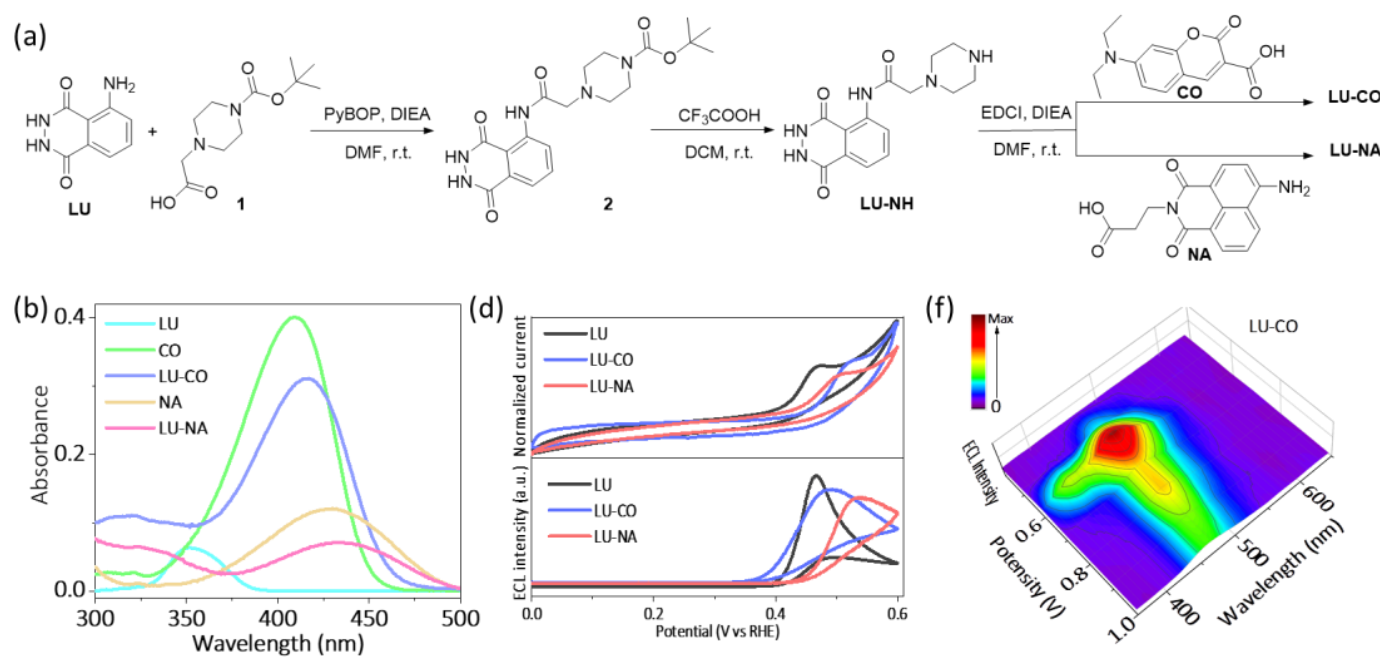

(c)
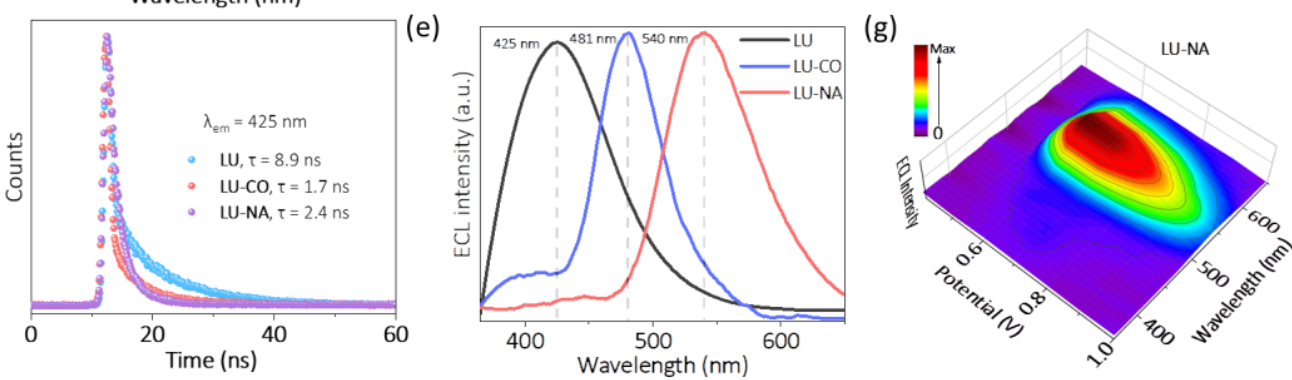

Figure 3. Synthesis, photophysical and ECL properties of LU-CO and LU-NA. (a) Brief synthetic routes of LU-CO and LU-NA. (b) UV-Vis absorption of LU-CO, LUNA, and the controls. (c) Time-resolved decay curves of LU, LU-CO, and LU-NA under $350 \mathrm{~nm}$ excitation in PBS $(0.01 \mathrm{M}, \mathrm{pH}=8.0)$. $\mathrm{CV}$ curves (top) and corresponding ECL intensity (down) of LU, LU-CO, and LU-NA (d). ECL emission spectra of $\mathbf{L U}$, LU-CO and LU-NA (e). Normalized ECL emission spectra changes of LU-CO (f) and LU-NA (g) at different potentials. Electrolytes: PBS (0.01M, pH = 8.0) with $\mathrm{H}_{2} \mathrm{O}_{2}(10$ $\mathrm{mM})$ as co-reactant.

The photophysical properties of LU-CO and LU-NA were investigated in phosphate buffer saline under ambient conditions. As shown in the UV-Vis absorption spectra (Figure 3b), since the amino group (electron-donating group) of LU was turned into amide group (electro-withdraw group), the absorption bands of the $\mathbf{L U}$ part in the $\mathbf{L U}$ CO and LU-NA were blue-shifted, while that of dye unit was slightly red-shifted due to the conversion of carboxyl group into amide group. The molar absorption coefficient of LU-CO and LU-NA was found to be different from that of the monomers (Table S7), consistent with the covalent conjugation between luminol and the dyes. The FL 
spectra in Figure S13 further showed that the maximum emission of LU-NA was almost identical to that of NA; while that of LU-CO was slightly red-shifted, compared with CO. It may attribute to the different electron-withdrawing abilities of carboxyl and amide groups. Interestingly, when excited at $350 \mathrm{~nm}$ (the maximum exciting wavelength of $\mathbf{L U}$ ), the fluorescence intensity of $\mathbf{L U}-\mathbf{C O}$ and $\mathbf{L U}-\mathbf{N A}$ was much higher than that of CO and NA (Figure S14), strongly suggesting the critical role of $\mathbf{L U}$ in photon absorption for the following intermolecular RET. More importantly, the FL lifetime $(\tau)$ of $\mathbf{L U}$ after conjugating with the $\mathbf{C O}$ and NA acceptors remarkedly decreased (Figure 3c), with an associated RET efficiency (ERET) of $81 \%$ and $73 \%$, respectively. All these experimental results confirmed that the effective RET from the $\mathbf{L U}$ donor unit to the dye acceptor units in LU-CO and LU-NA could occur under light irradiation. $^{58}$

We next exploited if RET in LU-CO and LU-NA could be driven and lighted up under electrogenic excitation. As shown in Figure 4a, LU, LU-CO, and LU-NA oxidized at almost the same peak potential of ca. $0.50 \mathrm{~V}$ under cyclic voltammetry in PBS with $\mathrm{H}_{2} \mathrm{O}_{2}$ as the co-reagent. Such oxidation could be assigned to the electron loss of the $\mathbf{L U}$ donor, ${ }^{59,60}$ as it was not observed for the single dyes (CO and NA) in the same condition (Figure S16). Simultaneously, LU-CO and LU-NA exhibited ECL signals from the starting of the electrochemical oxidation, and reached the maximum intensity at ca. $0.5 \mathrm{~V}$; while the dye monomers did not show any ECL signals (Figure S16). The ECL emission spectra of LU-CO and LU-NA were recorded to explore the ECL properties further. As shown in Figure 4b, except for the minor ECL emission of luminol at $425 \mathrm{~nm}, \mathbf{L U}-\mathbf{C O}$ and LU-NA showed a new ECL emission at $481 \mathrm{~nm}$ and $540 \mathrm{~nm}$, respectively, the profile of which was almost identical to that of the FL spectra (Figure S13). In contrast, no peaks at $481 \mathrm{~nm}$ or $540 \mathrm{~nm}$ were found under the same condition for the physically mixed dye (CO or NA) and LU (Figure S17), further verifying the occurrence of RET in the covalent linkage form. The efficiency of iECLRET in LU-CO and LU-NA, evaluated by ECL intensities (I) of the donor in the absence $\left(\mathrm{I}_{\mathrm{D}}\right)$ and in the presence $\left(\mathrm{I}_{\mathrm{DA}}\right)$ of the acceptor like that of FL (Figure S18), ${ }^{58}$ 
was approximated both as near-unity. These results evidently proved that the ECLinactive dyes were successfully lighted up by the efficient iECL-RET pathway.

Notably, typical excellent ECL properties of $\mathbf{L U}$ were well inherited in $\mathbf{L U} \mathbf{U} \mathbf{C O}$ and LU-NA. For instance, the ECL intensity of LU-CO and LU-NA could be modulated by the electrogenic excitation conditions, including the co-reagent concentration (Figure S20), the $\mathrm{pH}$ of aqueous solutions (Figure S21), and the electrochemical scanning rate (Figure S22). The ECL signals of LU-CO and LU-NA also exhibited high stability and reproducibility (Figure S23). Moreover, the 3D dynamic ECL emission plots of LU-CO and LU-NA at different potentials also strongly depended on LU (Figure 4c, 4d \& S19), e.g., the detectable ECL emission from ca. 0.4-0.5 V, and reached the maximum at ca. $0.6 \mathrm{~V}$. Therefore, considering the theoretical calculation, photophysical characterization, and the ECL properties, the ECL mechanism of LU-CO and LU-NA associated with iECL-RET was briefly described in Equation 1 \& 2 (see the detailed mechanism in Figure S24): ${ }^{61-63}$

$\mathrm{LU}^{-}$-dye $-\mathrm{e}^{-} \rightarrow \mathrm{LU}^{-\cdot}$-dye

$\mathrm{LU}^{-\cdot}$-dye $+\mathrm{O}_{2}^{-\cdot} \rightarrow \mathrm{LU}^{*}$-dye $\stackrel{\mathrm{RET}}{\longrightarrow} \mathrm{LU}-$ dye $^{*} \rightarrow \mathrm{LU}-$ dye $+\mathrm{h} v$

To prove the universality of our approach for lighting up ECL-inactive molecules, except for the blue LU-CO and yellow LU-NA, a series of ECL emitters with more refined color-tuning, was exploited by engineering electron-withdrawing substituents (Figure 4a). For this purpose, LU-NA-NO $\mathbf{O}_{2}$ was firstly synthesized (see characterization in Figure S25-28). Such $-\mathrm{NO}_{2}$ terminal group has a strong electronwithdrawing ability, making the intramolecular charge transfer (ICT) in the acceptor unit interdict (Figure 4a and S29). ${ }^{64,65}$ Consequently, it led to the interruption of iECLRET, and the ECL emission was mainly contributed by the $\mathbf{L U}$ donor. Interestingly, the nitro-group could be quantitatively reduced to amino-group with a much lower electron-withdrawing capacity by a facile enzymatic reaction using nitroreductase (NTR) and NADH. ${ }^{66-68}$ As a result, the ICT for the acceptor was improved, the iECLRET was boosted, and the ECL emission from the acceptor unit was enhanced. Along 
this line, the emission wavelength of LU-NA-X could be modulated by varying the concentration of NTR. As shown in Figure 4b, with the concentration of NTR increased from 25 to $200 \mu \mathrm{g} / \mathrm{mL}$, the maximum ECL emission wavelength was shifted significantly from $425 \mathrm{~nm}$ to $540 \mathrm{~nm}$. Therefore, based on the iECL-RET strategy, the color of ECL emitters could be modulated not only in a wide range by using different task-specific dyes but also in a refined manner by further harnessing substituents of different electronegativity (Figure 4c).

(a)

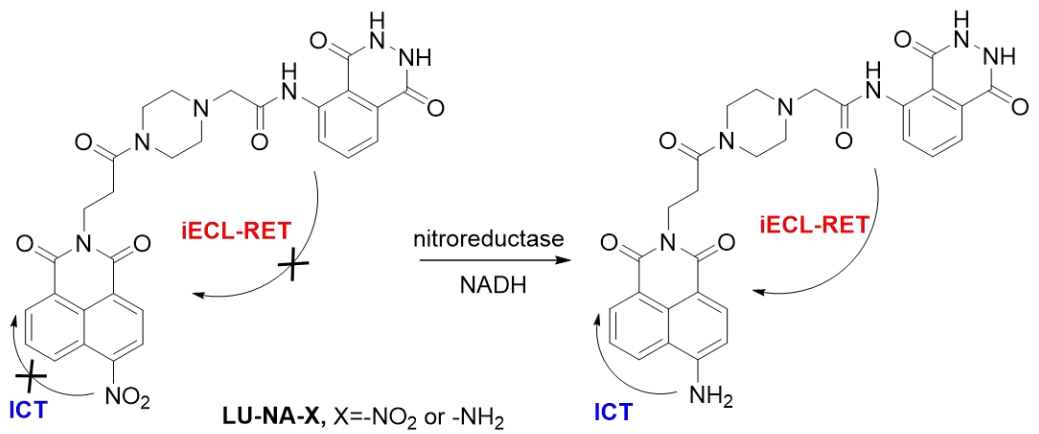

(b)

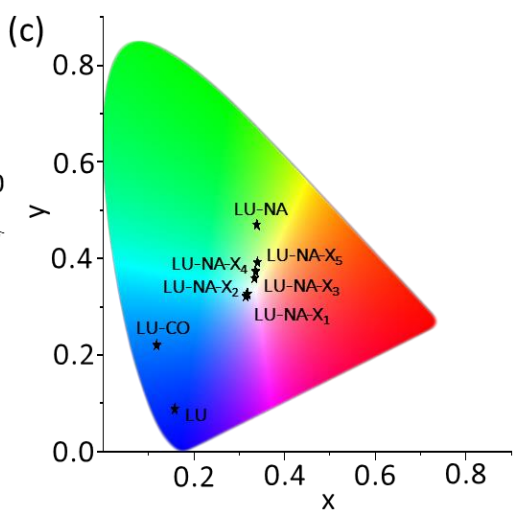

Figure 4. Generality of iECL-RET activation for a series of ECL emitters with fine-tuned properties. Enzymatic reduction conversion (a) and ECL emission spectra (b) of LU-NA-X with different amount of electron-withdrawing substituent. Color modulation emissions of LU, LU-CO, LU-NA, and a series of LU-NA-X in the CIE coordinate diagram (c).

It should be noted that the number of FL molecules with excellent photophysical properties is huge, but most of them are ECL inactive. The strategy of generating ECL signals from FL molecules through RET induced by an ECL initiator would provide an intriguing facile way to greatly expand the family members of ECL emitters. It would 
be highly envisioned that the well-developed sensing mechanism specifically for FL system could also be inherited by the proposed iECL-RET platform. For instance, upon the delicate correlation between LU-NA-X molecular structures and the unique ECL emission, a proposed example for sensitive and selective detection of NTR activities could also be constructed in turn. As shown in Figure S30, after the reaction between LU-NA-X and the analyte, the chromaticity coordinate showed a highly liner relationship to the concentration of NTR, while the other species were not included in the given range. Moreover, the proposed excited-state manipulation by RET to endow FL molecules with crossover luminesce is not only limited to ECL, but also promising for other type of luminesce, such as phosphorescence and X-ray fluorescence, which deserves future more fundamental and application studies.

\section{Conclusions}

In summary, a general iECL-RET strategy was proposed to facilely turn ECL inactive dyes into ECL activity emitters in aqueous phase. By screening the donors, acceptors, linkers and substituents according to the spectrum properties and theoretical prediction, a series of ECL emitters (LU-CO, LU-NA and LU-NA-X) were synthesized with a near unity RET efficiency and coarse/fine tunable emission wavelengths up to $115 \mathrm{~nm}$. Different to previous exploitation of new molecule single-handedly to address all the prerequisites of ECL, each unit in the proposed ECL ensemble performed maximally its own functions. Thanks to the generality of iECL-RET and huge number of dyes with diverse photophysical properties, this study would open a new era for developing ECL emitters and advance their multiformity and performances in more demanding prospective applications. Nonetheless, although the RET efficiency was close to unity, the overall ECL efficiency of LU-CO and LU-NA relative to $\mathbf{L U}$ was low (ca. 3\%) and need a future substantial improvement. 


\section{REFERENCES}

[1]. Zhifeng Ding, B. M. Q., Santosh K. Haram, Lindsay E. Pell, Brian A. Korgel, Allen J. Bard, Electrochemistry and Electrogenerated Chemiluminescence from Silicon Nanocrystal Quantum Dots. Science 2002, 296, 1293-1296.

[2]. Wu, P.; Hou, X.; Xu, J. J.; Chen, H. Y., Electrochemically generated versus photoexcited luminescence from semiconductor nanomaterials: bridging the valley between two worlds. Chem. Rev. 2014, $114(21), 11027-11059$.

[3]. Ma, X.; Gao, W.; Du, F.; Yuan, F.; Yu, J.; Guan, Y.; Sojic, N.; Xu, G., Rational Design of Electrochemiluminescent Devices. Acc. Chem. Res. 2021, 54 (14), 2936-2945.

[4]. Han, D.; Goudeau, B.; Manojlovic, D.; Jiang, D.; Fang, D.;

Sojic, N., Electrochemiluminescence Loss in Photobleaching. Angew. Chem. Int. Ed. 2021, 60 (14), 7686-7690.

[5]. Ding, H.; Zhou, P.; Fu, W.; Ding, L.; Guo, W.; Su, B., Spatially Selective Imaging of Cell-Matrix and Cell-Cell Junctions by Electrochemiluminescence. Angew. Chem. Int. Ed. 2021, 60 (21), 1176911773.

[6]. Qi, H.; Zhang, C., Electrogenerated Chemiluminescence Biosensing. Anal. Chem. 2019, 92 (1), 524-534.

[7]. Ma, C.; Cao, Y.; Gou, X.; Zhu, J.-J., Recent Progress in 
Electrochemiluminescence Sensing and Imaging. Anal. Chem. 2019, 92 (1), 431-454.

[8]. Richter, M. M., Electrochemiluminescence (ECL). Chem. Rev. 2004, 104 (6), 3003-3036.

[9]. Miao, W., Electrogenerated Chemiluminescence and Its Biorelated Applications. Chem. Rev. 2008, 108, 2506-2553.

[10]. Jae Il Kim, I.-S. S., Hasuck Kim, Jin-Kyu Lee, Efficient Electrogenerated Chemiluminescence from Cyclometalated Iridium(III) Complexes. J. Am. Chem. Soc. 2005, 127, 1614-1615.

[11]. Haghighatbin, M. A.; Laird, S. E.; Hogan, C. F., Electrochemiluminescence of cyclometalated iridium (III) complexes. Current Opinion in Electrochemistry 2018, 7, 216-223.

[12]. Yin, X.-B.; Dong, S.; Wang, E., Analytical applications of the electrochemiluminescence of tris (2,2' -bipyridyl) ruthenium and its derivatives. Trac-trend. Anal. Chem. 2004, 23 (6), 432-441.

[13]. Hesari, M.; Ding, Z., A Grand Avenue to Au Nanocluster Electrochemiluminescence. Acc. Chem. Res. 2017, 50 (2), 218-230.

[14]. Yang, L.; Zhang, B.; Fu, L.; Fu, K.; Zou, G., Efficient and Monochromatic Electrochemiluminescence of Aqueous-Soluble $\mathrm{Au}$ Nanoclusters via Host-Guest Recognition. Angew. Chem. Int. Ed. 2019, 58 (21), 6901-6905.

[15]. Ya-Lan Chang, R. E. P., Fu-Ren F. Fan, Allen J. Bard, and Paul F. 
Barbara, Electrogenerated Chemiluminescence of Single Conjugated Polymer Nanoparticles. J. Am. Chem. Soc. 2008, 130, 8906-8907.

[16]. Chen, S.; Ma, H.; Padelford, J. W.; Qinchen, W.; Yu, W.; Wang, S.; Zhu, M.; Wang, G., Near Infrared Electrochemiluminescence of Rod-Shape 25-Atom AuAg Nanoclusters That Is Hundreds-Fold Stronger Than That of Ru(bpy)3 Standard. J. Am. Chem. Soc. 2019, 141 (24), 9603-9609.

[17]. Zinna, F.; Voci, S.; Arrico, L.; Brun, E.; Homberg, A.; Bouffier, L.; Funaioli, T.; Lacour, J.; Sojic, N.; Di Bari, L., Circularly-Polarized Electrochemiluminescence from a Chiral Bispyrene Organic Macrocycle. Angew. Chem. Int. Ed. 2019, 58 (21), 6952-6956.

[18]. Shen, M.; Zhu, X. H.; Bard, A. J., Electrogenerated chemiluminescence of solutions, films, and nanoparticles of dithienylbenzothiadiazole-based donor-acceptor-donor red fluorophore. Fluorescence quenching study of organic nanoparticles. J. Am. Chem. Soc. 2013, 135 (24), 8868-8873.

[19]. Qi, H.; Zhang, C.; Huang, Z.; Wang, L.; Wang, W.; Bard, A. J., Electrochemistry and Electrogenerated Chemiluminescence of 1,3,5Tri(anthracen-10-yl)-benzene-Centered Starburst Oligofluorenes. J. Am. Chem. Soc. 2016, 138 (6), 1947-1954.

[20]. Guo, W.; Ding, H.; Gu, C.; Liu, Y.; Jiang, X.; Su, B.; Shao, Y., Potential-Resolved Multicolor Electrochemiluminescence for 
Multiplex Immunoassay in a Single Sample. J. Am. Chem. Soc. 2018, 140 (46), 15904-15915.

[21]. Peng, H.; Huang, Z.; Sheng, Y.; Zhang, X.; Deng, H.; Chen, W.; Liu, J., Pre-oxidation of Gold Nanoclusters Results in a $66 \%$ Anodic Electrochemiluminescence Yield and Drives Mechanistic Insights. Angew. Chem. Int. Ed. 2019, 58 (34), 11691-11694.

[22]. Doeven, E. H.; Zammit, E. M.; Barbante, G. J.; Francis, P. S.; Barnett, N. W.; Hogan, C. F., A potential-controlled switch on/off mechanism for selective excitation in mixed electrochemiluminescent systems. Chem. Sci. 2013, 4 (3), 977-982.

[23]. Doeven, E. H.; Zammit, E. M.; Barbante, G. J.; Hogan, C. F.; Barnett, N. W.; Francis, P. S., Selective excitation of concomitant electrochemiluminophores: tuning emission color by electrode potential. Angew. Chem. Int. Ed. 2012, 51 (18), 4354-7.

[24]. Haghighatbin, M. A.; Lo, S.-C.; Burn, P. L.; Hogan, C. F., Electrochemically tuneable multi-colour electrochemiluminescence using a single emitter. Chem. Sci. 2016, 7 (12), 6974-6980.

[25]. Han, Z.; Yang, Z.; Sun, H.; Xu, Y.; Ma, X.; Shan, D.; Chen, J.; Huo, S.; Zhang, Z.; Du, P.; Lu, X., Electrochemiluminescence Platforms Based on Small Water-Insoluble Organic Molecules for Ultrasensitive Aqueous-Phase Detection. Angew. Chem. Int. Ed. 2019, 58 (18), 5915-5919. 
[26]. Wei, X.; Zhu, M. J.; Cheng, Z.; Lee, M.; Yan, H.; Lu, C.; Xu,

J. J., Aggregation-Induced Electrochemiluminescence of Carboranyl Carbazoles in Aqueous Media. Angew. Chem. Int. Ed. 2019, 58 (10), 31623166.

[27]. Carrara, S.; Aliprandi, A.; Hogan, C. F.; De Cola, L., Aggregation-Induced Electrochemiluminescence of Platinum(II) Complexes. J. Am. Chem. Soc. 2017, 139 (41), 14605-14610.

[28]. Zhang, Y.; Zhao, Y.; Han, Z.; Zhang, R.; Du, P.; Wu, Y.; Lu, X., Switching the Photoluminescence and Electrochemiluminescence of Liposoluble Porphyrin in Aqueous Phase by Molecular Regulation. Angew. Chem. Int. Ed. 2020, 59 (51), 23261-23267.

[29]. Peng, H. P.; $\quad$ Huang, Z. N.; $\quad$ Deng, H. H.; Wu, W. H.; Huang, K. Y.; Li, Z. L.; Chen, W.; Liu, J. W., Dual Enhancement of Gold Nanocluster Electrochemiluminescence: Electrocatalytic Excitation and Aggregation-Induced Emission. Angew. Chem. Int. Ed. 2020, 59 (25), 9982-9985.

[30]. Zhu, D.; Zhang, Y.; Bao, S.; Wang, N.; Yu, S.; Luo, R.; Ma, J.; Ju, H.; Lei, J., Dual Intrareticular Oxidation of Mixed-Ligand Metal-Organic Frameworks for Stepwise Electrochemiluminescence. $J$. Am. Chem. Soc. 2021, 143 (8), 3049-3053.

[31]. Voci, S.; Duwald, R.; Grass, S.; Hayne, D. J.; Bouffier, L.; Francis, P. S.; Lacour, J.; Sojic, N., Self-enhanced multicolor 
electrochemiluminescence by competitive electron-transfer processes. Chem. Sci. 2020, 11 (17), 4508-4515.

[32]. Carrara, S.; Arcudi, F.; Prato, M.; De Cola, L., Amine-Rich Nitrogen-Doped Carbon Nanodots as a Platform for Self-Enhancing Electrochemiluminescence. Angew. Chem. Int. Ed. 2017, 56 (17), 47574761.

[33]. Wang, T.; Wang, D.; Padelford, J. W.; Jiang, J.; Wang, G., NearInfrared Electrogenerated Chemiluminescence from Aqueous Soluble Lipoic Acid Au Nanoclusters. J. Am. Chem. Soc. 2016, 138 (20), 63806383.

[34]. Jin, Z.; Zhu, X.; Wang, N.; Li, Y.; Ju, H.; Lei, J., Electroactive Metal - Organic Frameworks as Emitters for Self - Enhanced Electrochemiluminescence in Aqueous Medium. Angew. Chem. Int. Ed. 2020, 59 (26), 10446-10450.

[35]. Tan, X.; Zhang, B.; Zou, G., Electrochemistry and Electrochemiluminescence of Organometal Halide Perovskite Nanocrystals in Aqueous Medium. J. Am. Chem. Soc. 2017, 139 (25), 8772-8776.

[36]. Guo, W.; Ding, H.; Zhou, P.; Wang, Y.; Su, B., Electrochemiluminescence Waveguide in Single Crystalline Molecular Wires. Angew. Chem. Int. Ed. 2020, 59 (17), 6745-6749.

[37]. Hesari, M.; Swanick, K. N.; Lu, J. S.; Whyte, R.; Wang, S.; 
Ding, Z., Highly Efficient Dual-Color Electrochemiluminescence from BODIPY-Capped PbS Nanocrystals. J. Am. Chem. Soc. 2015, 137 (35), $11266-9$

[38]. Wong, J. M.; Zhang, R.; Xie, P.; Yang, L.; Zhang, M.; Zhou, R.; Wang, R.; Shen, Y.; Yang, B.; Wang, H. B.; Ding, Z., Revealing Crystallization-Induced Blue-Shift Emission of a Di-Boron Complex by Enhanced Photoluminescence and Electrochemiluminescence. Angew. Chem. Int. Ed. 2020, 59 (40), 17461-17466.

[39]. Irkham; Fiorani, A.; Valenti, G.; Kamoshida, N.; Paolucci, F.; Einaga, Y., Electrogenerated Chemiluminescence by in Situ Production of Coreactant Hydrogen Peroxide in Carbonate Aqueous Solution at a BoronDoped Diamond Electrode. J. Am. Chem. Soc. 2019, 142 (3), 1518-1525. [40]. Wang, N.; Gao, H.; Li, Y.; Li, G.; Chen, W.; Jin, Z.; Lei, J.; Wei, Q.; Ju, H., Dual Intramolecular Electron Transfer for In Situ Coreactant - Embedded Electrochemiluminescence Microimaging of Membrane Protein. Angew. Chem. Int. Ed. 2020, 60 (1), 197-201.

[41]. Lv, Y.; Chen, S.; Shen, Y.; Ji, J.; Zhou, Q.; Liu, S.; Zhang, Y., Competitive Multiple-Mechanism-Driven Electrochemiluminescent Detection of 8-Hydroxy-2'-deoxyguanosine. J. Am. Chem. Soc. 2018, 140 (8), 2801-2804.

[42]. Zhao, T.; Zhou, Q.; Lv, Y.; Han, D.; Wu, K.; Zhao, L.; Shen, Y.; Liu, S.; Zhang, Y., Ultrafast Condensation of Carbon Nitride on 
Electrodes with Exceptional Boosted Photocurrent and

Electrochemiluminescence. Angew. Chem. Int. Ed. 2019, 59 (3), 1139-1143.

[43]. Zanut, A.; Fiorani, A.; Canola, S.; Saito, T.; Ziebart, N.;

Rapino, S.; $\quad$ Rebeccani, S.; $\quad$ Barbon, A.; Irie, T.; Josel, H.-P.; Negri,

F.; Marcaccio, M.; Windfuhr, M.; Imai, K.; Valenti, G.; Paolucci, F., Insights into the mechanism of coreactant electrochemiluminescence facilitating enhanced bioanalytical performance. Nat. Commun. 2020, 11

[44]. Irkham; Fiorani, A.; Valenti, G.; Kamoshida, N.; Paolucci, F.; Einaga, Y., Electrogenerated Chemiluminescence by in Situ Production of Coreactant Hydrogen Peroxide in Carbonate Aqueous Solution at a BoronDoped Diamond Electrode. J. Am. Chem. Soc. 2020, 142 (3), 1518-1525. [45]. Wang, F.; Lin, J.; Zhao, T.; Hu, D.; Wu, T.; Liu, Y., Intrinsic "Vacancy Point Defect" Induced Electrochemiluminescence from Coreless Supertetrahedral Chalcogenide Nanocluster. J. Am. Chem. Soc. 2016, 138 (24), 7718-7724.

[46]. Zhang, J.; Jin, R.; Jiang, D.; Chen, H.-Y., Electrochemiluminescence-Based Capacitance Microscopy for Label-Free Imaging of Antigens on the Cellular Plasma Membrane. J. Am. Chem. Soc. 2019, 141 (26), 10294-10299.

[47]. Maar, R. R.; Z Zhang, R.; Stephens, D. G.; Ding, Z.; Gilroy, J. B., Near-Infrared Photoluminescence and Electrochemiluminescence from a 
Remarkably Simple Boron Difluoride Formazanate Dye. Angew. Chem. Int. Ed. 2019, 58 (4), 1052-1056.

[48]. Rizzo, F.; Polo, F.; Bottaro, G.; Fantacci, S.; Antonello, S.; Armelao, L.; Quici, S.; Maran, F., From Blue to Green: Fine-Tuning of Photoluminescence and Electrochemiluminescence in Bifunctional Organic Dyes. J. Am. Chem. Soc. 2017, 139 (5), 2060-2069.

[49]. Walter, E. R. H.; Ge, Y.; Mason, J. C.; Boyle, J. J.; Long, N. J., A Coumarin-Porphyrin FRET Break-Apart Probe for Heme Oxygenase-1. J. Am. Chem. Soc. 2021, 143 (17), 6460-6469.

[50]. Wu, L.; Huang, C.; Emery, B. P.; Sedgwick, A. C.; Bull, S. D.; He, X.-P.; Tian, H.; Yoon, J.; Sessler, J. L.; James, T. D., Förster resonance energy transfer (FRET)-based small-molecule sensors and imaging agents. Chem. Soc. Rev. 2020, 49 (15), 5110-5139.

[51]. Nakanotani, H.; Higuchi, T.; Furukawa, T.; Masui, K.; Morimoto, K.; Numata, M.; Tanaka, H.; Sagara, Y.; Yasuda, T.; Adachi, C., High-efficiency organic light-emitting diodes with fluorescent emitters. Nat. Commun. 2014, 5 (1).

[52]. Lu, H.-J.; Xu, J.-J.; Zhou, H.; Chen, H.-Y., Recent advances in electrochemiluminescence resonance energy transfer for bioanalysis: Fundamentals and applications. Trac-trend. Anal. Chem. 2020, 122.

[53]. Ye, J.; Zhu, L.; Yan, M.; Zhu, Q.; Lu, Q.; Huang, J.; Cui, H.; Yang, X., Dual-Wavelength Ratiometric Electrochemiluminescence 
Immunosensor for Cardiac Troponin I Detection. Anal. Chem. 2018, 91 (2), $1524-1531$.

[54]. Dong, Y.-P.; Gao, T.-T.; Zhou, Y.; Zhu, J.-J., Electrogenerated

Chemiluminescence Resonance Energy Transfer between Luminol and CdSe@ZnS Quantum Dots and Its Sensing Application in the Determination of Thrombin. Anal. Chem. 2014, 86 (22), 11373-11379.

[55]. Eliel, E. L., Conformational Analysis in Mobile Cyclohexane Systems. Angew. Chem. Int. Ed. 1965, 4 (9), 761-774.

[56]. Jensen, F. R.; Bushweller, C. H., Conformational Preferences in Cyclohexanes and Cyclohexenes. In Advances in Alicyclic Chemistry, 1971; Vol. 3, pp 139-194.

[57]. Algar, W. R.; Hildebrandt, N.; Vogel, S. S.; Medintz, I. L., FRET as a biomolecular research tool — understanding its potential while avoiding pitfalls. Nat. Methods 2019, 16 (9), 815-829.

[58]. Valeur, B. B.-S., M. N., Chapter 8: Excitation Energy Transfer. In Molecular Fluorescence, 2nd Edition;. Wiley-VCH: Singapore: 2013; p $213-262$.

[59]. Liu, X.; Qi, W.; Gao, W.; Liu, Z.; Zhang, W.; Gao, Y.; Xu, G., Remarkable increase in luminol electrochemiluminescence by sequential electroreduction and electrooxidation. Chem. Commun. 2014, 50 (93), 14662-14665.

[60]. Qiao, Y.; Li, Y.; Fu, W.; Guo, Z.; Zheng, X., Enhancing the 
Electrochemiluminescence of Luminol by Chemically Modifying the Reaction Microenvironment. Anal. Chem. 2018, 90 (15), 9629-9636.

[61]. Kitte, S. A.; Gao, W.; Zholudov, Y. T.; Qi, L.; Nsabimana, A.; Liu, Z.; Xu, G., Stainless Steel Electrode for Sensitive Luminol Electrochemiluminescent Detection of $\mathrm{H} 2 \mathrm{O} 2$, Glucose, and Glucose Oxidase Activity. Anal. Chem. 2017, 89 (18), 9864-9869.

[62]. Garcia-Segura, S.; Centellas, F.; Brillas, E., Unprecedented Electrochemiluminescence of Luminol on a Boron-Doped Diamond ThinFilm Anode. Enhancement by Electrogenerated Superoxide Radical Anion. J. Phys. Chem. C 2012, 116 (29), 15500-15504.

[63]. Mayer, M.; Takegami, S.; Neumeier, M.; Rink, S.; Jacobi von Wangelin, A.; Schulte, S.; Vollmer, M.; Griesbeck, A. G.; Duerkop, A.; Baeumner, A. J., Electrochemiluminescence Bioassays with a WaterSoluble Luminol Derivative Can Outperform Fluorescence Assays. Angew. Chem. Int. Ed. 2018, 57 (2), 408-411.

[64]. Brennecke, B.; $\quad$ Wang, Q.; Zhang, Q.; Hu, H. Y.; Nazaré, M., An Activatable Lanthanide Luminescent Probe for Time-Gated Detection of Nitroreductase in Live Bacteria. Angew. Chem. Int. Ed. 2020, 59 (22), $8512-8516$

[65]. Karan, S.; Cho, M. Y.; Lee, H.; Lee, H.; Park, H. S.; Sundararajan, M.; Sessler, J. L.; Hong, K. S., Near-Infrared Fluorescent Probe Activated by Nitroreductase for In Vitro and In Vivo Hypoxic Tumor 
Detection. J. Med. Chem. 2021, 64 (6), 2971-2981.

[66]. Yoon, S. A.; Chun, J.; Kang, C.; Lee, M. H., Self-Calibrating Bipartite Fluorescent Sensor for Nitroreductase Activity and Its Application to Cancer and Hypoxic Cells. ACS Appl. Bio Mater. 2020, 4 (3), 2052-2057.

[67]. Zhang, S.; Chen, H.; Wang, L.; Qin, X.; Jiang, B.-P.; Ji, S.C.; Shen, X.-C.; Liang, H., A General Approach to Design Dual Ratiometric Fluorescent and Photoacoustic Probe for Quantitatively Visualizing Tumor Hypoxia Levels in Vivo. Angew. Chem. Int. Ed. 2021. [68]. Sarkar, S.; ～Lee, H.; Ryu, H. G.; Singha, S.; Lee, Y. M.; Reo, Y. J.; Jun, Y. W.; Kim, K. H.; Kim, W. J.; Ahn, K. H., A Study on Hypoxia Susceptibility of Organ Tissues by Fluorescence Imaging with a Ratiometric Nitroreductase Probe. ACS Sens. 2020, 6 (1), 148-155. 